\title{
Second moment closure near the two-component limit
}

\author{
By Robert Rubinstein, ${ }^{1}$ Sharath S. Girimaji ${ }^{2}$ \\ ${ }^{1}$ Computational Aerosciences Branch, NASA Langley Research Center, Hampton, VA 23681, \\ USA \\ ${ }^{2}$ Department of Aerospace and Mechanical Engineering, Texas A\&M University, College \\ Station, TX USA
}

(Received ?? and in revised form ??)

In turbulence modeling, the two-component limit is defined by $\left\langle u_{3} u_{3}\right\rangle=0$, or more generally by $\left\langle u_{i} u_{j}\right\rangle \xi_{i} \xi_{j}=0$ for some vector $\xi_{i}$ (here and throughout, $u_{i}$ denotes velocity fluctuations). Although this limit certainly represents a rather extreme state of turbulence, it is approached in several practically important problems: for example, in near-wall turbulence, $\left\langle u_{3} u_{3}\right\rangle \ll\left\langle u_{2} u_{2}\right\rangle \approx\left\langle u_{1} u_{1}\right\rangle$. The two-component limit is also connected to the realizability problem: the condition that a predicted Reynolds stress tensor $R_{i j}$ can be represented as the correlation $R_{i j}=\left\langle u_{i} u_{j}\right\rangle$ for some real stochastic field $u_{i}$ is equivalent to the positivity condition $R_{i j} \xi_{i} \xi_{j} \geq 0$ for arbitrary real indeterminates $\xi_{i}$ (Schumann (1977)). If a stress field becomes unrealizable, so that $R_{i j} \xi_{i}^{*} \xi_{j}^{*}<0$ for some vector $\xi_{i}^{*}$, it obviously must do so by passing through a two-component state in which $R_{i j} \xi_{i}^{*} \xi_{j}^{*}=0$. Formulating conditions on models that insure realizability a priori must therefore consider the behavior of models at or near the two-component limit (Shih and Lumley (1985)). Because of these considerations, the two-component limit has been given considerable attention in the modeling literature (Sjögren and Johansson (2000)).

The purpose of this paper is to explore some wider implications of the two-component limit for both single-point turbulence models and spectral closure theories. Although the two-component limit arises most naturally in inhomogeneous problems like wall-bounded turbulence, the analysis will be restricted to homogeneous turbulence. But since homogeneous turbulence is the crucial case for realizability (Girimaji (2004)), the conclusions will nevertheless be applicable to modeling.

The essential point of our argument is that whereas the evolution of the stochastic velocity field is Markovian because it is governed by the Navier-Stokes equations, the exact stress evolution equation is not Markovian because it is unclosed. This property of moment evolution has been stressed by Kraichnan (1959). We will show that modeling stress evolution at the two-component limit with a closure that is Markovian in the stresses alone leads to basic inconsistencies in single-point modeling and, perhaps surprisingly, in spectral models as well.

Turbulence transport modeling begins by assuming that the fluctuating velocity field can be described adequately by some finite number of basic statistics, for example, by the energy and dissipation rate in a two-equation model (Jones and Launder (1972)), or by the dissipation rate and the components of the stress tensor in a second moment closure (Hanjalić and Launder (1972)). Whilst such assumptions about the kinematics of turbulence are well satisfied in a very broad class of turbulent flows, turbulence modeling requires a much stronger assumption about dynamics, namely that these basic statistics satisfy closed equations of motion. However, no fundamental considerations justify this 
assumption, which is instead supported after the fact by comparing model predictions with experimental measurements or numerical data.

This paper proposes a critical assessment of this type of closure assumption in the important special case of second moment closure. We begin with the exact Reynolds stress transport equation in the form

$$
\dot{R}_{i j}=P_{i j}-\Phi_{i j}-\epsilon_{i j}+T_{i j}
$$

The notation and terminology of (Girimaji (2004)) are used: the dot denotes the convective derivative with respect to the mean flow, $R_{i j}$ is the Reynolds stress, $P_{i j}$ is the production, $\Phi_{i j}$ is the pressure-strain correlation, $\epsilon_{i j}$ the dissipation tensor, and $T_{i j}$ the transport. As noted earlier, in second moment closure, the basic statistics are the stress components and the dissipation rate; all other correlations will be expressed in terms of them and the mean velocity. The production is an explicit function of the stress itself and does not require closure. We will consider homogeneous turbulence, in which the transport term $T_{i j}$ vanishes; thus, the pressure-strain correlation $\Phi_{i j}$ is the only unclosed term.

Second moment closure assumes the functional dependence

$$
\Phi=\mathrm{f}(\mathrm{R}, \nabla \mathbf{U}, \epsilon)
$$

where $\Phi$ denotes the pressure-strain correlation tensor, R the Reynolds stress tensor, and $\nabla \mathbf{U}$ the mean velocity gradient: index-free notation has been introduced in order to emphasize that the left side is a tensor function of the arguments of $\mathrm{f}$. This representation includes the possibility of a term of the form $A(\mathrm{R}, \nabla \mathbf{U}, \epsilon) \mathrm{I}$ where I is the identity matrix and $A$ is a function of the joint scalar invariants of $\mathrm{R}$ and $\nabla \mathbf{U}$ and of $\epsilon$.

We will show that Eq. (0.2) is inadequate in the two-component limit. The reason is that the pressure-strain correlation dynamics makes the evolution of the stresses nonMarkovian. However, substitution of Eq. (0.2) in Eq. (0.1) results in a Markovian model for the stress evolution. Although a Markovian model of a non-Markovian physical phenomenon is never desirable, this simplification of the dynamics will prove to have particularly dire implications in the two-component limit: in this limit, Eq. (0.2) yields a mathematically incomplete representation of the pressure-strain correlation leading to qualitatively inadmissable behavior, regardless of the functional form assumed for $\mathrm{f}$.

For this demonstration, we consider initially two-component turbulence for which $R_{33}(0)=R_{13}(0)=R_{23}(0)=0$. The exact equation for $R_{33}$ is

$$
\dot{R}_{33}=-2\left\langle u_{3} u_{p}\right\rangle \frac{\partial U_{3}}{\partial x_{p}}-2\left\langle u_{3} \frac{\partial p}{\partial x_{3}}\right\rangle-2 \nu\left\langle\frac{\partial u_{3}}{\partial x_{p}} \frac{\partial u_{3}}{\partial x_{p}}\right\rangle
$$

and since all correlations on the right side vanish initially, also

$$
\dot{R}_{33}(0)=0
$$

Because every correlation in Eq. (0.3) contains a factor $u_{3}$, it might appear that a permanent two-component state in which $u_{3} \equiv 0$ is a solution. We will prove that instead, the Navier-Stokes equations drive the solution away from the two-component state. To rule out direct linear couplings that trivially generate nonzero $u_{3}$, suppose that if a mean velocity gradient exists, it is nonzero only in the 1-2 plane. Then

$$
\frac{\partial U_{3}}{\partial x_{i}}=\frac{\partial U_{i}}{\partial x_{3}}=0
$$

It will be convenient to say that the Reynolds stress and mean velocity gradient are two-component tensors. In this case, the production term on the right side of Eq. (0.3) 
vanishes leaving

$$
\dot{R}_{33}=-2\left\langle u_{3} \frac{\partial p}{\partial x_{3}}\right\rangle-2 \nu\left\langle\frac{\partial u_{3}}{\partial x_{p}} \frac{\partial u_{3}}{\partial x_{p}}\right\rangle
$$

The first correlation on the right side of Eq. (0.6) satisfies

$$
\left.\frac{d}{d t}\left\langle u_{3} \frac{\partial p}{\partial x_{3}}\right\rangle\right|_{t=0}=-\left\langle\frac{\partial p}{\partial x_{3}}(\mathbf{x}, 0) \frac{\partial p}{\partial x_{3}}(\mathbf{x}, 0)\right\rangle
$$

whereas the second correlation satisfies

$$
\frac{d}{d t}\left\langle\frac{\partial u_{3}}{\partial x_{p}}(\mathbf{x}, 0) \frac{\partial u_{3}}{\partial x_{p}}(\mathbf{x}, 0)\right\rangle=0
$$

Therefore,

$$
\ddot{R}_{33}(0)=2\left\langle\frac{\partial p}{\partial x_{3}}(\mathbf{x}, 0) \frac{\partial p}{\partial x_{3}}(\mathbf{x}, 0)\right\rangle
$$

The two-component limit certainly permits $\left.\left\langle\left(\partial p / \partial x_{3}\right)^{2}\right\rangle\right|_{t=0} \neq 0$. Therefore,

$$
\ddot{R}_{33}(0)>0
$$

This result, with Eq. (0.4) implies that $R_{33}(t)>0$ for sufficiently small positive $t$. Thus, the Navier-Stokes dynamics drive the solution away from the initial two-component state. Note that Eq. (0.10) states the 'strong realizability' condition (Shih and Lumley (1985), Girimaji (2004)).

Now consider the predictions of second moment closure for this problem. Standard representation theorems for tensor functions (Pope (2000)) applied to Eq. (0.2) give

$$
\dot{\mathrm{R}}=A_{0} \mathrm{I}+\sum_{1 \leq n \leq N} A_{n} \mathrm{C}^{n}
$$

where the $\mathrm{C}^{n}$ are an appropriate basis of tensors that can be formed from $\mathrm{R}$ and $\nabla \mathbf{U}$, and the $A_{m}$ with $0 \leq m \leq N$ are functions of scalar invariants of $\mathrm{R}$ and $\nabla \mathbf{U}$. In the case of no mean flow, the basis tensors $\mathrm{C}^{n}$ consist of $\mathrm{R}$ and its powers. $\dagger$ All of the powers $\mathrm{R}^{n}$ are twocomponent for $n \geq 1$ because $\mathrm{R}$ is, and $A_{0}$ must vanish when $t=0$ because $\dot{R}_{33}(0)=0$. It follows that $\dot{R}(0)$ must be two-component if there is no mean flow. If there is a nonzero mean gradient, the basis $\mathrm{C}^{n}$ will also contain products of $\mathbf{R}$ and $\nabla \mathbf{U}$. However, since $\nabla \mathbf{U}$ has been assumed to be two-component (Eq. (0.5)), all of these products are also two-component when $t=0$. It again follows that $\dot{\mathrm{R}}(0)$ is two-component.

If $\dot{R}(0)$ is two-component, then

$$
\dot{R}_{13}(0)=\dot{R}_{23}(0)=\dot{R}_{33}(0)=0
$$

This conclusion is easily shown to be inconsistent with the equations of motion, which imply that

$$
\dot{R}_{13}(0)=\left\langle\dot{u}_{1}(0) u_{3}(0)\right\rangle+\left\langle u_{1}(0) \dot{u}_{3}(0)\right\rangle
$$

Although the first correlation vanishes, the second need not vanish in general. Thus, the assumed second moment closure form Eq. (0.2) is inconsistent with Navier-Stokes dynamics. This inconsistency leads to an even more serious problem.

Consider the evolution of the 'non-two-component' stresses $R_{13}, R_{23}$, and $R_{33}$ under Eq. (0.11). Because $\nabla \mathbf{U}$ is two-component, some of the $C^{n}$ might happen to be twocomponent for arbitrary R. Such terms will not contribute to the evolution of any of $R_{13}$,

$\dagger$ In fact, the Cayley-Hamilton theorem implies that the explicit representation depends only on powers up to the second, but this fact is not essential to the argument. 
$R_{23}$, or $R_{33}$. But in general, the $\mathrm{C}^{n}$ will not be identically two-component. Eq. (0.12) requires that each term $A_{n} \mathrm{C}^{n}$ be two-component when $t=0$ for arbitrary values of $R_{11}(0), R_{12}(0)$, and $R_{13}(0)$. Consider the Taylor series expansion of $A_{n} C_{i 3}^{n}$ for $i=1,2,3$ in powers of the stress components. Each term in the expansion must contain either $R_{13}$, $R_{23}$, or $R_{33}$ to at least the first power, otherwise $A_{n} C_{i j}^{n}$ could only be two-component when $t=0$ for special choices of $R_{11}(0), R_{12}(0)$, and $R_{22}(0)$. We conclude that

$$
A_{n} C_{i 3}^{n}=\alpha_{13}^{i n} R_{13}+\alpha_{23}^{i n} R_{23}+\alpha_{33}^{i n} R_{33} \text { for } i=1,2,3
$$

where the $\alpha^{\text {in }}$ are functions of the components of $\mathbf{R}$ and $\nabla \mathbf{U}$.

Substituting the results Eq. (0.14) in Eq. (0.11), it is seen that under the closure hypothesis Eq. (0.2), compliance with the initial conditions dictated by the equations of motion requires that the evolution equations for $R_{13}, R_{23}$, and $R_{33}$ have the special form

$$
\begin{aligned}
& \dot{R}_{13}=A_{11} R_{13}+A_{12} R_{23}+A_{13} R_{33} \\
& \dot{R}_{23}=A_{21} R_{13}+A_{22} R_{23}+A_{23} R_{33} \\
& \dot{R}_{33}=A_{31} R_{13}+A_{32} R_{23}+A_{33} R_{33}
\end{aligned}
$$

where the quantities $A_{i j}$ are functions of the components of $\mathrm{R}$ and $\nabla \mathbf{U}$ and of $\epsilon$. Regardless of the analytical expressions for the $A_{i j}, R_{i 3}(t) \equiv 0$ satisfies Eq. (0.15) with the initial conditions $R_{i 3}(0)=0$; thus, the closure assumption of Eq. (0.2) implies that the solution to the model equations never leaves the two-component state. Thus, Eq. (0.2) is inconsistent with the equations of motion, which imply that the two-component state cannot persist. The problems of modeling near the two-component limit have been analyzed by Craft and Launder (2002), who investigate the kinematic constraints imposed by the vanishing of certain components of the pressure-strain tensor at this limit. The present work complements this investigation by considering the dynamics of evolution away from a two-component state.

The essential point can be illustrated in a simple model problem. Suppose that the stochastic variable $x$ satisfies

$$
\dot{x}=a
$$

where $a$ is a known stochastic process. Then

$$
\frac{d}{d t}\left\langle x^{2}\right\rangle=2\langle a x\rangle
$$

Suppose that $x(0)=0$. As in the stress equation, $x \equiv 0$ is not a solution of the complete hierarchy of moment equations that begins with Eq. (0.17), because

$$
\left.\frac{d^{2}}{d t^{2}}\left\langle x^{2}\right\rangle\right|_{t=0}=\left.2\langle\dot{a} x\rangle\right|_{t=0}+\left.2\left\langle a^{2}\right\rangle\right|_{t=0}=\left.2\left\langle a^{2}\right\rangle\right|_{t=0}
$$

need not vanish. But if we introduce a closure hypothesis that

$$
\langle a x\rangle=F\left(\left\langle x^{2}\right\rangle\right)
$$

so that

$$
\frac{d}{d t}\left\langle x^{2}\right\rangle=F\left(\left\langle x^{2}\right\rangle\right)
$$

with $F(0)=0$ imposed to respect the initial conditions, a differentiable $F$ forces the conclusion that any solution for $\left\langle x^{2}\right\rangle$ that vanishes initially must in fact vanish identically. The problem is that since under Eq. (0.16), $x \sim t$ for short times if $x(0)=0,\left\langle x^{2}\right\rangle \sim t^{2}$ for short times, but $\langle a x\rangle \sim t$. Since $t$ is not a smooth function of $t^{2}$ near $t=0$, the 
closure assumption in Eq. (0.19) cannot be correct. Arguing that, in view of the shorttime behavior, we should set $\langle a x\rangle \sim \sqrt{\left\langle x^{2}\right\rangle}$, the result is a singular differential equation $(d / d t)\left\langle x^{2}\right\rangle \sim \sqrt{\left\langle x^{2}\right\rangle}$, for which the solution satisfying $\left\langle x^{2}\right\rangle(0)=0$ is not unique.

The analysis for the stress tensor is essentially the same, although the presence of tensor components necessarily generates the more elaborate argument leading to Eq. (0.15). The common features are the Markovian evolution of $x$, Eq. (0.16), but the non-Markovian evolution of the moment $\left\langle x^{2}\right\rangle$ due to its unclosed evolution equation Eq. (0.17). The closure hypothesis Eq. (0.19), like Eq. (0.2), is inconsistent with the short-time behavior of $\langle a x\rangle$ and $\left\langle x^{2}\right\rangle$.

This argument is restricted to the short-time properties of initially two-component turbulence. The demonstration that $R_{33}$ grows is consistent with Kolmogorov's ideas, according to which turbulence should become more isotropic as it evolves. We would like to supplement these short-time results with long-time results available from integrating the equations of rapid distortion theory (RDT) (Hunt and Carruthers (1990)). In the standard Fourier representation, these equations have the form

$$
\dot{Q}_{i j}(\mathbf{k}, t)=\Pi_{i j}(\mathbf{k}, t)+\Phi_{i j}(\mathbf{k}, t)
$$

where

$$
Q_{i j}(\mathbf{k}, t)=\int d \mathbf{r} \exp [i \mathbf{k} \cdot \mathbf{r}]\left\langle u_{i}(\mathbf{x}+\mathbf{r}, t) u_{j}(\mathbf{x}, t)\right\rangle
$$

is the Fourier transform of the two-point correlation tensor,

$$
\Pi_{i j}(\mathbf{k}, t)=Q_{i p}(\mathbf{k}, t) \partial U_{p} / \partial x_{j}+Q_{j p}(\mathbf{k}, t) \partial U_{p} / \partial x_{i}
$$

is the production tensor, and

$$
\Phi_{i j}(\mathbf{k}, t)=i\left\langle p(-\mathbf{k}, t)\left[k_{j} u_{i}(\mathbf{k}, t)+k_{i} u_{j}(\mathbf{k}, t)\right]\right\rangle
$$

is the pressure-strain tensor in which the pressure $p$ is evaluated in the linear approximation as

$$
p(\mathbf{k}, t)=k^{-2} k_{m} u_{n}(\mathbf{k}, t) \partial U_{n} / \partial x_{m}
$$

The Reynolds stress is expressed in terms of the correlation by

$$
R_{i j}(t)=\int d \mathbf{k} Q_{i j}(\mathbf{k}, t)
$$

The important fact is that the RDT equations are closed evolution equations for $Q_{i j}(\mathbf{k}, t)$ because $\Phi_{i j}(\mathbf{k}, t)$ can be expressed explicitly in terms of $Q_{i j}(\mathbf{k}, t)$ through Eqs. $(0.24)$ and (0.25). Of course, the integrated quantities $R_{i j}(t)$ and

$$
\Phi_{i j}(t)=\int d \mathbf{k} \Phi_{i j}(\mathbf{k}, t)
$$

do not satisfy closed equations; the 'rapid pressure-strain' model formulated as Eq. (0.2) attempts to express $\Phi_{i j}$ in terms of $R_{i j}$, the mean gradients, and the dissipation rate. RDT is therefore a good vantage point from which to evaluate this hypothesis.

Figure 1 shows the evolution of $R_{11}(t), R_{22}(t), R_{33}(t)$, and $R_{12}(t)$ according to RDT, starting from a two-component $Q_{i j}(\mathbf{k}, 0)$ under mean shear $\partial U_{1} / \partial x_{2}$. The main conclusion is that $R_{33}(t)$ does indeed become nonzero; at large times, it is actually larger than $R_{22}$. Figure 2 shows the evolution of $\Phi_{11}(t), \Phi_{22}(t), \Phi_{33}(t)$, and $\Phi_{12}(t)$. This figure pinpoints the reason that Eq. (0.2) fails. Obviously $\Phi_{33}(t) \sim t$ at short times, whereas $R_{33}(t) \sim t^{2}$. These results can be compared to the model problem described earlier. As in that problem, second moment closure tries to express a quantity which grows linearly in time as a smooth function of a quantity which grows quadratically. 
RDT shows that the coupling of the stress components to the rapid pressure-strain tensor sustains long-time growth of $R_{33}(t)$. We would like to reconsider homogeneous anisotropic turbulence without mean shear from the viewpoint of spectral closure theory in order to understand the nonlinear mechanisms which lead to long-time growth of $R_{33}(t)$.

In a classic paper, Herring (1974) studied the relaxation of axisymmetric turbulence using the direct interaction approximation (DIA) of Kraichnan (1959). We quote the relevant equations as

$$
\begin{aligned}
& \dot{\phi}^{\lambda}\left(\mathbf{k} ; t, t^{\prime}\right)=-\nu k^{2} \phi^{\lambda}\left(\mathbf{k} ; t, t^{\prime}\right)+\sum_{\mu, \nu} \int d \mathbf{p} d \mathbf{q} \delta(\mathbf{k}-\mathbf{p}-\mathbf{q}) \times \\
& \left\{A_{\lambda \mu \nu}(\mathbf{k}, \mathbf{p}, \mathbf{q}) \int_{0}^{t^{\prime}} d s g^{\lambda}(\mathbf{k} ; t, s) \phi^{\mu}(\mathbf{p} ; t, s) \phi^{\nu}(\mathbf{q} ; t, s)\right. \\
& \left.+B_{\lambda \mu \nu}(\mathbf{k}, \mathbf{p}, \mathbf{q}) \int_{0}^{t} d s g^{\mu}(\mathbf{p} ; t, s) \phi^{\lambda}(\mathbf{k} ; t, s) \phi^{\nu}(\mathbf{q} ; t, s)\right\}
\end{aligned}
$$

In this equation, $\lambda, \mu, \nu \in\{1,2\}$; they are not tensor indices, so any summations involving them are indicated explicitly. $\phi^{1}\left(\mathbf{k} ; t, t^{\prime}\right)$ and $\phi^{2}\left(\mathbf{k} ; t, t^{\prime}\right)$ are scalar descriptors of an axisymmetric two-time correlation tensor in the elementary but elaborate kinematics of (Herring (1974)); isotropy is the special case $\phi^{1}=\phi^{2}$ with dependence on $k=|\mathbf{k}|$ only, and the two-component state corresponds to $\phi^{2}=0$. The quantities $A_{\lambda \mu \nu}$ and $B_{\lambda \mu \nu}$ are geometric factors. It is crucial to our argument that $A_{\lambda \mu \nu} \geq 0$; the $B_{\lambda \mu \nu}$ are typically negative, and represent damping effects. Finally, $g^{\lambda}(\mathbf{k} ; t, s)$ are additional quantities linked to the temporal decorrelation of turbulence; we will only use the initial conditions $g^{\lambda}(\mathbf{k} ; 0,0)=1$.

Herring (1974) shows by explicit calculation that initially two-component axisymmetric turbulence becomes fully three-component under the DIA evolution equations (see Figure 9 of (Herring (1974)). The reason that DIA predicts the growth of $\phi^{2}\left(\mathbf{k} ; t, t^{\prime}\right)$ starting from $\phi^{2}(\mathbf{k} ; 0,0)=0$ is that the $A_{211}$ term in Eq. $(0.28)$ couples $\phi^{2}\left(\mathbf{k} ; t, t^{\prime}\right)$ to $\phi^{1}\left(\mathbf{k} ; t, t^{\prime}\right)$. The short-time form of the evolution equation for $\phi^{2}\left(\mathbf{k} ; t, t^{\prime}\right)$ is

$$
\dot{\phi}^{2}\left(\mathbf{k} ; t, t^{\prime}\right)=t^{\prime} \int d \mathbf{p} d \mathbf{q} \delta(\mathbf{k}-\mathbf{p}-\mathbf{q}) A_{211}(\mathbf{k}, \mathbf{p}, \mathbf{q}) \phi^{\mu}(\mathbf{p} ; 0,0) \phi^{\nu}(\mathbf{q} ; 0,0)
$$

Thus, $\phi^{2}\left(\mathbf{k} ; t, t^{\prime}\right) \sim t t^{\prime}$ and the single-time correlation grows as $\phi^{2}(\mathbf{k}, t)=\phi^{2}(\mathbf{k}, t, t) \sim t^{2}$. This short-time quadratic growth is consistent with earlier conclusions based on shorttime properties of the Navier-Stokes equations. Note also that Eq. (0.29) shows that $\ddot{\phi}^{2}(\mathbf{k}, 0)>0$.

We would like to highlight the ingredients in DIA which make departure from an initial two-component state possible: one way is to ask to what extent DIA can be simplified and yet remain consistent with this property. Suppose that we introduce the 'almostMarkovian' (Kraichnan (1971)) modification

$$
\begin{aligned}
& \frac{d \phi^{\lambda}}{d t}(\mathbf{k}, t)=-\nu k^{2} \phi^{\lambda}(\mathbf{k}, t)+\sum_{\mu, \nu} \int d \mathbf{p} d \mathbf{q} \delta(\mathbf{k}-\mathbf{p}-\mathbf{q}) \times \\
& \left\{A_{\lambda \mu \nu}(\mathbf{k}, \mathbf{p}, \mathbf{q}) \Theta_{\lambda \mu \nu}(\mathbf{k}, \mathbf{p}, \mathbf{q} ; t) \phi^{\mu}(\mathbf{p}, t) \phi^{\nu}(\mathbf{q}, t)\right. \\
& \left.-B_{\lambda \mu \nu}(\mathbf{k}, \mathbf{p}, \mathbf{q}) \Theta_{\mu \lambda \nu}(\mathbf{k}, \mathbf{p}, \mathbf{q} ; t) \phi^{\lambda}(\mathbf{k}, t) \phi^{\nu}(\mathbf{q}, t)\right\}
\end{aligned}
$$

where $\phi^{\lambda}(\mathbf{k}, t)=\phi^{\lambda}(\mathbf{k}, t, t)$ and the time-scales $\Theta_{\lambda \mu \nu}(\mathbf{k}, t)$ satisfy evolution equations 
with the structure (Kraichnan (1971))

$$
\dot{\Theta}_{\lambda \mu \nu}(\mathbf{k}, \mathbf{p}, \mathbf{q} ; t)=1-\left[\eta_{\lambda}(\mathbf{k}, t)+\eta_{\mu}(\mathbf{p}, t)+\eta_{\nu}(\mathbf{q}, t)\right] \Theta_{\lambda \mu \nu}(\mathbf{k}, \mathbf{p}, \mathbf{q} ; t)
$$

with suitable damping factors $\eta$. The combination of Eqs. (0.30) and (0.31), which depends on single-time quantities alone, is a multiple state variable Markovian model like the test-field model (Kraichnan (1971)); Kraichnan calls such models 'almost Markovian' to distinguish them from models like EDQNM (Orszag (1973)) which are Markovian in the correlations alone.

The short-time properties of this simpler model agree with those of Eq. (0.28): if, in a two-component initial state, we may assume that $\Theta_{\lambda \mu \nu}$ vanishes unless $\lambda=\mu=\nu=1$, then we will have $\Theta_{211} \sim t$ for short times, and consequently $\phi^{2} \sim t^{2}$. But it is critical to this argument that $\Theta_{211}$ evolve independently of $\Theta_{111}$; the plausible attempt to simplify the model by using a single relaxation time $\Theta$ would lose these short-time properties. Despite its complexity, such a model would also predict the persistence of an initial two-component state.

Another plausible but unsatisfactory simplification is to construct a theory that is Markovian in the correlations alone, for example, by replacing Eq. (0.31) by its long-time limit $\Theta_{\lambda \mu \nu}(\mathbf{k}, \mathbf{p}, \mathbf{q} ; t)=1 /\left[\eta_{\lambda}(\mathbf{k}, t)+\eta_{\mu}(\mathbf{p}, t)+\eta_{\nu}(\mathbf{q}, t)\right]$. Regardless of how the damping factors $\eta$ are chosen, this theory cannot be made consistent with the short-time growth $\phi^{2} \sim t^{2}$ : given that the Navier Stokes equations will require $\dot{\phi}^{2}(\mathbf{k}, 0)=0$, this theory theory will force $\phi^{2}$ to vanish for all times. We conclude that (fully) Markovian spectral closure for axisymmetric turbulence is inconsistent with the equations of motion for twocomponent initial conditions. The minimal spectral closure model that is consistent with the properties of two-component turbulence is no more than 'almost-Markovian.' Timescale evolution cannot be avoided, moreover, a complete matrix of time-scales must be evolved; a single scalar time-scale is insufficient. It should be noted that RDT can escape the two-component state using the correlation tensor alone because coupling to the mean field by the pressure-strain correlation makes the stress evolution non-Markovian.

Although we have demonstrated that the closure hypothesis of Eq. (0.2) is incorrect only in a very special circumstance, this demonstration underscores that such hypotheses are uncontrolled approximations of uncertain validity. Perhaps more attention should be given to finding conditions under which these hypotheses can actually be justified.

In conclusion, we would like to summarize some implications of this analysis for the realizability problem for single-point closures, which first motivated this investigation. We have found that at the two-component limit, the strong realizability condition $\ddot{R}_{33}(0)>0$ or its spectral analog: $\ddot{Q}_{33}(\mathbf{k}, 0)>0$ and $\ddot{\phi}^{2}(\mathbf{k}, 0)>0$, is satisfied, whether as a consequence of short time analysis of single-point correlations, RDT, or DIA. To this extent, our results support the strong realizability constraint as a realistic statement of dynamics near the two-component limit. However, we also find that the standard second moment closure assumption Eq. (0.2) is not consistent with this constraint, since it forces $\ddot{R}_{33}(0)=0$. It is significant that Markovianization of DIA also leads to the unsatisfactory conclusion that $\ddot{\phi}^{2}(\mathbf{k}, 0)=0$.

The strong realizability constraint is therefore physically correct, but cannot be consistently enforced with the usual Markovian formulation of second moment closure. We believe that Markovianization of the time dependence underlies an important unresolved issue in rapid pressure-strain correlation modeling: fundamental constraints on these models are (i) that the closure be linear in the stresses, and (ii) that the closure satisfy the strong realizability condition. To date, no model satisfies both conditions, and it has been claimed that strong realizability requires nonlinear models. At the level of Marko- 
vian second moment closure, the weak realizability condition (Pope (2000)), which makes the two-component limit inaccessible, therefore seems unavoidable.

We would like to briefly outline some alternate approaches to the realizability problem suggested by the present analysis. One way to make a single-point model consistent with the two-component limit is to extend it, for example to include the components of the pressure-strain correlation as independent dynamic variables; for shear turbulence, the structure tensors (Kassinos et al. (2000)) are also reasonable candidates. This type of extension of the model can be compared formally to Kraichnan's almost-Markovianization: the introduction of a new variable abandons the Markovian character of Eq. (0.2) and allows $\dot{R}$ to be a functional of the time history of the arguments of $f$.

Another possibility is reformulate the problem so that stress is not the basic variable. For example, (Girimaji (2004)) suggests that the realizability of the Reynolds stress can be guaranteed by ensuring the realizability of the closure models for each individual term in the exact stress transport equation. This approach shifts the enforcement of realizability away from the troublesome two-component limit. A related approach is to introduce a symmetric tensor $S$ with the property that $R=S^{2}$. If the theory is expressed near the two-component limit in terms of $\mathrm{S}$ rather than $\mathrm{R}$, then a Markovian model for $\mathrm{S}$ will allow $\mathrm{S} \sim t$ near the two-component state, yet $\mathrm{R} \sim t^{2}$ as required. The introduction of $S$ effectively regularizes the problem near the two-component limit. It is critical that the theory is expressed in terms of $S$ and not $R$ : we are not suggesting the introduction of a matrix square root, which would lead to interminable problems of sign and the strong possibility of non-uniqueness in the time evolution.

\section{REFERENCES}

Craft, T. J., And Launder, B. E. 2002 Closure modelling near the two-component limit in Launder, B. E. and Sandham, N. D. (editors), Closure strategies for turbulent and transitional flows, Cambridge University Press, 2002, pp. 102-126.

Girimaji, S. S. 2004 A new perspective on realizability of turbulence models J. Fluid Mech. 512, 191-210.

HANJALIĆ, K. AND LAUNDER, B. E. 1972 Reynolds stress model of turbulence and its application to thin shear flows J. Fluid Mech. 52, 609.

HeRring, J. R. 1974 Approach of axisymmetric turbulence to isotropy Phys. Fluids 17, 859872.

Hunt, J. C. R. And CARruthers, D. J. 1990 Rapid distortion theory and the 'problems' of turbulence J. Fluid Mech. 212, 497-532.

Jones, W. P. AND LAunder, B. E. 1972 The prediction of laminarization with a two-equation model of turbulence, Int. J. Heat Mass Transf. 15, 301-314.

Kassinos, S. C., Reynolds, W. C., And Rogers, M. M. 2000 One-point turbulence structure tensors J. Fluid Mech. 428, 213-248.

Kraichnan, R. H. 1959 The structure of isotropic turbulence at very high Reynolds numbers J. Fluid Mech. 5, 497-543.

Kraichnan, R. H. 1971 An almost-Markovian Galilean-invariant turbulence model J. Fluid Mech. 47, 513-524.

Lumley, J. L. 1983 Turbulence modeling J. App. Mech. 50, 1097-1103.

OrszaG, S. A. 1973 Lectures on the statistical theory of turbulence, in R. Balian \& J.-L. Preube, Fluid Dynamics, Gordon and Breach, New York.

Pope, S. B. 2000 Turbulent flows, Cambridge University Press.

Schumann, U. 1977 Realizability of Reynolds-stress turbulence models Phys. Fluids 20, 721725.

ShiH, T.-H., AND Lumley, J. L. 1985 Modeling of pressure correlation terms in Reynolds stress and scalar flux equations, Cornell University Technical Report FDA-85-3. 
SJögren, T., AND Johansson, A. V. 2000, Development and calibration of nonlinear algebraic models for terms in the Reynolds stress transport equations, Phys. Fluids 12, 1554-1572. 


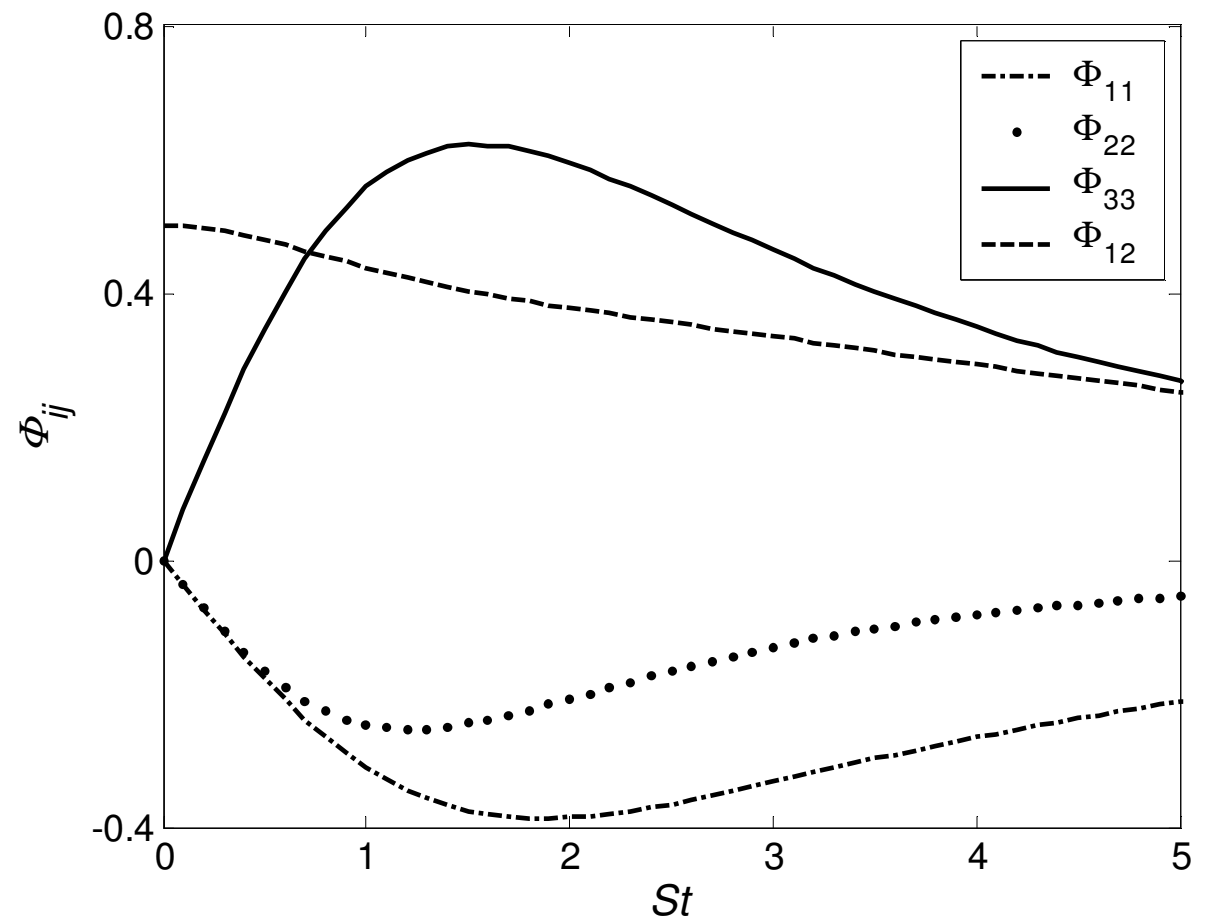

Pressure-rate-of-strain tensor components

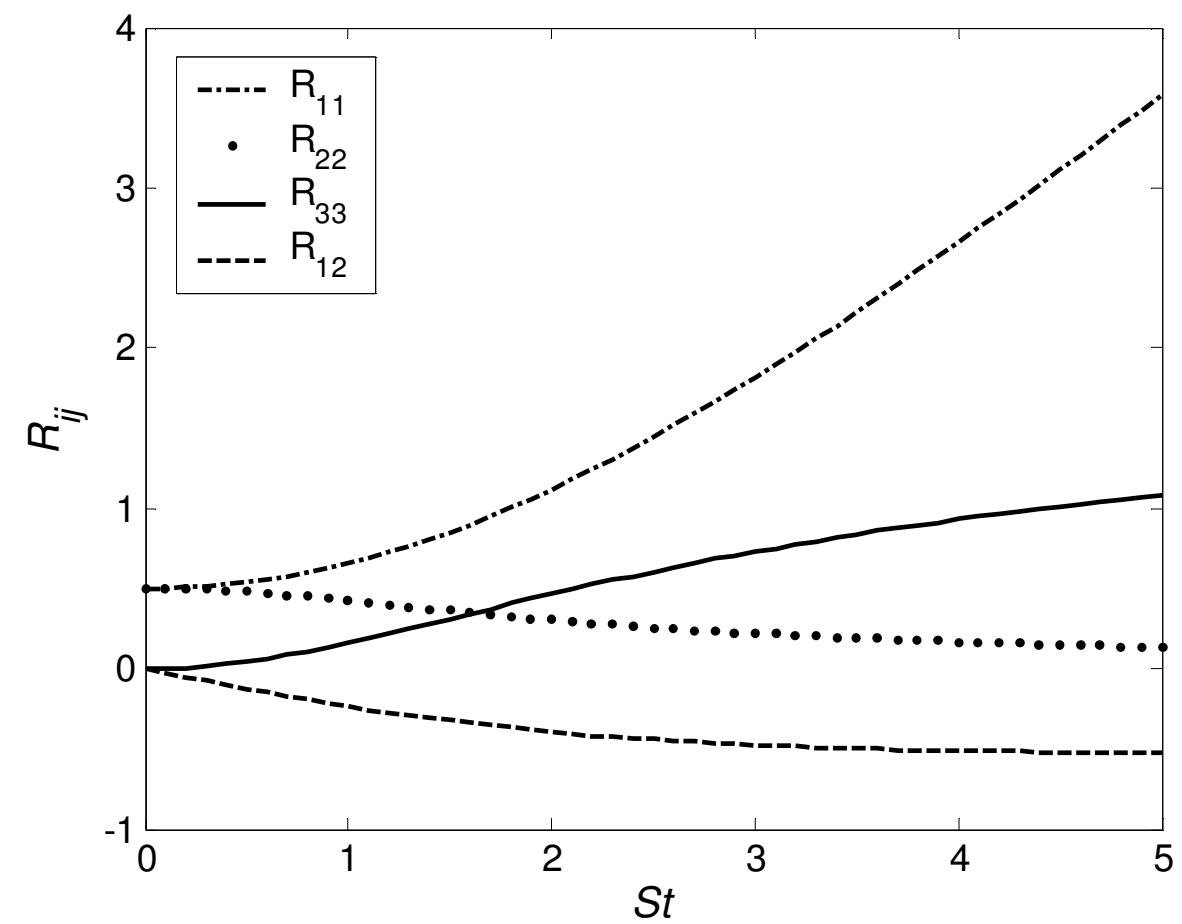

Reynolds stress tensor components 